[4] A. Maccari,Vibration control for the primary resonance of a cantilever beam by a time delay state feedback, JSV,259(2), (2003), 241-251

[5] A. Maccari, Vibration amplitude control for a van der Pol-Duffing oscillator with time delay, JSV, 317, (2008), 20-29

[6] A. Maccari, Arbitrary amplitude periodic solutions for parametrically excited systems with time delay, Nonlinear Dyn,51, (2008),111-126

[7] T. M. Morrison, R. H. Rand, 2:1 Resonance in the delayed nonlinear Mathieu equation, Nonlinear Dyn, 50, (2007), 341-352

[8] S. M., Sah, R. H. Rand, Using delay to quench undesirable vibrations, Nonlinear Dyn, 62, (2010), 407-416

[9] S. Shao, K. M. Masri, M. I. Younis, The effect of time-delayed feedback controller on an electrically actuated resonator, Nonlinear Dyn, 74, (2013), 257-270

[10] A. M. Elnaggar, K. M. Khalil, The response of nonlinear controlled system under an external excitation via time delay state feedback, Journal of King Saud University - Engineering Sciences, 28, (2016),75-83

[11] K. A. Alhazza, M. F. Daqaq, A. H. Nayfeh, D. J. Inman, Nonlinear vibrations of parametrically excited cantilever beams subjected to non-linear delayed-feedback control, International Journal of Non-Linear Mechanics, 43, (2008), $801-812$

[12] K. A. Alhazza, M. A. Majeed, Free vibrations control of a cantilever beam using combined time delay feedback, JVC,18(5), (2011), 609-621

[13] M. F. Daqaq, K. A. Alhazza, Y. Qaroush, On primary resonances of weakly nonlinear delay systems with cubic nonlinearities, Nonlinear Dyn, 64, (2011), 253-277

[14] Ling Xu, Lei Chen, Weili Xiong, Parameter estimation and controller design for dynamic systems from the step responses based on the Newton, Nonlinear Dyn, 79, (2015), 2155-2163

[15] Ling $\mathrm{Xu}, \mathrm{A}$ proportional differential control method for a time-delay system using the Taylor expansion approximation, Applied Mathematics and Computation 236, (2014, 391-399

[16] N. A. Saeed, W. A. El-Ganaini, Time-delayed control to suppress the nonlinear vibrations of a horizontally suspended Jeffcott-rotor system, Applied Mathematical Modelling, 44, (2017), 523-539

[17] N. A. Saeed, W. A. El-Ganaini, Utilizing time-delays to quench the nonlinear vibrations of a two-degree-offreedom system, Meccanica, doi: 10.1007/s11012-017-0643-z

[18] Y. Ishida, T. Inoue, Vibration Suppression of nonlinear rotor systems using a dynamic damper, JVC, 138, (2007),1127-1143

[19] A. Nayfeh, D. Mook, Nonlinear Oscillations. Wiley, New York (1995).

[20] L.F. Shampine, S. Thompson, Solving DDEs in MATLAB. Applied Numerical Mathematics, 37, (2001), $441-458$ 


\title{
SOME COMPLEX DYNAMICAL BEHAVIORS OF THE NEW 6D FRACTIONAL-ORDER HYPERCHAOTIC LORENZ-LIKE SYSTEM
}

\author{
Ahmed A. M. Farghaly ${ }^{1}$ and A. A.-H. Shoreh ${ }^{2}$. \\ ${ }^{1}$ Department of Mathematics, Faculty of Science, Assiut University, Assiut 71516, Egypt. \\ ${ }^{2}$ Department of Mathematics, Faculty of Science, Al-Azhar University, Assiut, 71524, Egypt.
}

E-mail: aafarghaly166@hotmail.com, ahmed.shoreh@yahoo.com

\begin{abstract}
In this paper, we introduce the fractional version of the new $6 \mathrm{D}$ hyperchaotic Lorenz-like system which has been introduced recently in the literature. Hyperchaotic behaviors of higher order increase the randomness and higher unpredictability of the corresponding system. Some complex dynamical behaviors such as hyperchaotic of order 5, Poincaré mapping and bifurcation diagram are analyzed and investigated. The stability region of the new fractional-order system is investigated. The values of the fractional-order and the system parameters at which hyperchaotic attractors exist are calculated based on the sign of their Lyapunov exponents. The complete synchronization of the hyperchaotic attractors of order 5 is studied. A scheme is stated to derive the analytical formula of the control function to study this kind of synchronization. An excellent agreement is found upon comparison this analytical formula with numerical experiments.

Keywords: Fractional calculus, chaotic, hyperchaotic, bifurcation, synchronization.

PACS numbers: 05.45.-a, 05.45.Pq, 05.45.Xt
\end{abstract}

\section{Introduction}

For nearby 50 years, the chaotic theory was an interesting phenomenon of dynamical systems. It has been found to be very useful and has great potential in many disciplines such as the fields of communication, Laser, neural network, nonlinear circuits, and etc [1-6]. Famous examples of chaotic systems are Lorenz [3], Rössler [4], Chen [5] and Lü [6]. Chaotic behaviors have been widely studied on a great numbers of integer order models. In the literature, the fractional calculus is increasingly used as effective tools to model many systems. For instance, in physics 
fractional derivatives have been widely used in the mathematical modeling of viscoelastic materials $[7,8]$, some electromagnetic problems are described using fractional integro-differentiation operators [9], The anomalous diffusion phenomena in inhomogeneous media can be explained by non integer derivative based on equations of diffusion $[10,11]$, the resistance-capacitanceinductance (RLC) interconnect model of a transmission line is a fractional-order model [12], heat conduction as a dynamical process can be more adequately modeled by fractional-order models than by their integer order counterparts [13]. In biology, it has been deduced that the membranes of cells of biological organism have fractional-order electrical conductance [14]. In economics, it is known that some finance systems can display fractional-order dynamics [15]. More examples for fractional-order dynamics can be found in [16-21].

Studying the fractional-order systems has attracted increasing attention for instance, in [23] it has been shown that a limit cycle can be generated in the fractional-order Wien bridge oscillator, existence of a limit cycle for the fractional Brusselator has been shown in [24]. Also, it has been found that some fractional-order differential systems can demonstrate chaotic behavior, such as Duffing's oscillators, Chua circuit, jerk model, Lorenz system, Lü system, Chen system and Rössler system [25-32]. Hyperchaotic system is usually defined as a system with at least two positive Lyapunov exponents which can increase the randomness and higher unpredictability of the corresponding system. It is believed that chaotic systems with higher-dimensional attractors have much wider applications. Recently, some authors studied the fractional-order hyperchaotic system by adding a nonlinear terms in fractional-order systems. Wu et al. found that hyperchaotic behaviors exist in the fractional-order hyperchaotic Chen system with the order as low as 3.72 [32]. The lowest order for hyperchaos in fractional-order Rössler system is 3.8 [33].

Synchronization of chaotic fractional-order differential systems starts to attract increasing attention due to its potential applications in secure communication, image processing, chemical and biological systems, information science and control processing [34]. There are many methods of synchronization, such as complete synchronization [35], lag synchronization [36], projective synchronization [37], generalization of combination-combination synchronization [38], phase synchronization [39] and so on.

The rest of this paper is organized as follows: Section 2, contains some basic concepts of fractional calculus and the new 6D fractional-order hyperchaotic Lorenz-like system is introduced. In section 3, the dynamical behaviors of this system are studied. We calculated the values of the fractional-order and the system parameters at which chaotic and hyperchaotic exist based on the sign of their Lyapunov exponents. This system has hyperchaotic attractors of order 2, 3, 4 and 5. Bifurcation diagrams are plotted. In section 4, A scheme is presented to study the complete synchronization of the hyperchaotic attractors of our system. An analytical formula is derived to study this kind of synchronization. In the last section, we summarize and discuss our results. 


\section{Basic concepts and fractional system model}

Fractional calculus is a generalization of integration and differentiation to a non integer-order. The integro-differential operator $D_{t}^{\alpha}$ defined by

$$
D_{t}^{\alpha}= \begin{cases}\frac{d^{\alpha}}{d t^{\alpha}}, & \alpha>0 ; \\ 1, & \alpha=0 ; \\ \int_{0}^{t}(d \xi)^{-\alpha}, & \alpha<0 .\end{cases}
$$

There are many definitions for fractional derivatives [17]. The commonly used definitions are Riemann-Liouville and Caputo. The Riemann-Liouville fractional derivative, is given by

$$
\left.\left.{ }_{c} D_{t}^{\alpha} f(t)=\frac{1}{\Gamma(n-\alpha)} \frac{d^{n}}{d t^{n}} \int_{c}^{t} \frac{f(\xi)}{(t-\xi)^{\alpha-n+1}} d \xi, \quad t>c, \quad \operatorname{Re}(\alpha) \in\right] n-1, n\right] .
$$

The Caputo differential operator is given by

$$
\left.\left.{ }_{c} D_{t}^{\alpha} f(t)=\frac{1}{\Gamma(n-\alpha)} \int_{c}^{t} \frac{f^{(n)}(\xi)}{(t-\xi)^{\alpha-n+1}} d \xi, \quad t>c, \quad \alpha \in\right] n-1, n\right],
$$

where $\Gamma($.$) is the gamma function. The merit of the Caputo definition is the \alpha$-order derivative of a constant is zero.

Recently, Yang et al. [40] presented a new 6D hyperchaotic Lorenz-Like system with four positive Lyapunov exponents which can be described as:

$$
\begin{aligned}
& \dot{\mathbf{x}}=a(\mathbf{y}-\mathbf{x})+\mathbf{u}, \\
& \dot{\mathbf{y}}=c \mathbf{x}-\mathbf{y}-\mathbf{x z}+\mathbf{v}, \\
& \dot{\mathbf{z}}=-b \mathbf{z}+\mathbf{x y} \\
& \dot{\mathbf{u}}=d \mathbf{u}-\mathbf{x z} \\
& \dot{\mathbf{v}}=-k \mathbf{y} \\
& \dot{\mathbf{w}}=h \mathbf{w}+l \mathbf{y}
\end{aligned}
$$

where $a b h k l \neq 0, a, b, c$ and $h$ are parameters, $l$ is the coupling parameter, $d$ and $k$ are two control parameters. When $(a, b, c, d, k, h, l)=(10,8 / 3,28,2,8.4,1,1)$, Yang et al. proved that system (4) has a unique unstable equilibrium point $E_{0}(0,0,0,0,0,0)$ and the six Lyapunov exponents which are $\left(\lambda_{1}, \lambda_{2}, \lambda_{3}, \lambda_{4}, \lambda_{5}, \lambda_{6}\right)=(0.9995,0.4892,0.3256,0.0514,0.0000,-12.5324)$. 
In this paper, we aim to introduce the fractional version of system (4) which can be written as follows:

$$
\begin{aligned}
& { }_{c} D_{t}^{\alpha} \mathbf{x}(t)=a(\mathbf{y}-\mathbf{x})+\mathbf{u}, \\
& { }_{c} D_{t}^{\alpha} \mathbf{y}(t)=c \mathbf{x}-\mathbf{y}-\mathbf{x z}+\mathbf{v}, \\
& { }_{c} D_{t}^{\alpha} \mathbf{z}(t)=-b \mathbf{z}+\mathbf{x y}, \\
& { }_{c} D_{t}^{\alpha} \mathbf{u}(t)=d \mathbf{u}-\mathbf{x z}, \\
& { }_{c} D_{t}^{\alpha} \mathbf{v}(t)=-k \mathbf{y}, \\
& { }_{c} D_{t}^{\alpha} \mathbf{w}(t)=h \mathbf{w}+l \mathbf{y} .
\end{aligned}
$$

Here, ${ }_{c} D_{t}^{\alpha}$ is $\alpha$-order Caputo differential operator and $\alpha$ is the derivative order of the corresponding state variables $\mathbf{x}, \mathbf{y}, \ldots, \mathbf{w}$. When $\alpha=1$, system (5) becomes the $6 \mathrm{D}$ hyperchaotic Lorenz-Like system (4), so the fractional-order version can be seen as a generalization of the integral order system. Now, we will study the dynamical behaviors of system (5).

\section{Dynamics of system (5)}

In this section, we show that system (5) exhibit hyperchaotic solutions and calculate numerically the values of the fractional derivative order and the parameters at which these solutions exist. In our numerical studies, we used Adams-Bashforth-Moulton method [41] to find the numerical solution of system (5). To calculate the Lyapunov exponents $\lambda_{j}, j=1,2, \ldots, 6$ of this system we used the Wolf algorithm [42]. The signs of Lyapunov exponents provide a good classification of the solutions of this system. Mahmoud et al. [43] stated these classifications, see Table 1.

Table 1. The signs of Lyapunov exponents and the corresponding type of solutions.

\begin{tabular}{ccccccc}
\hline$\lambda_{1}$ & $\lambda_{2}$ & $\lambda_{3}$ & $\lambda_{4}$ & $\lambda_{5}$ & $\lambda_{6}$ & Dynamics \\
\hline- & - & - & - & - & - & Solutions approach fixed point \\
0 & - & - & - & - & - & Periodic solution (limit cycles) \\
0 & 0 & - & - & - & - & Quasi-periodic solution (2-torus) \\
0 & 0 & 0 & - & - & - & Quasi-periodic solution (3-torus) \\
+ & - & - & - & - & - & Chaotic solution \\
+ & + & - & - & - & - & Hyperchaotic solution of order 2 \\
+ & + & + & - & - & - & Hyperchaotic solution of order 3 \\
+ & + & + & + & - & - & Hyperchaotic solution of order 4 \\
+ & + & + & + & + & - & Hyperchaotic solution of order 5 \\
\hline
\end{tabular}

The fractional Lyapunov dimension of the attractors for system (5) according to the Kaplan- 
Yorke [43] conjecture is defined as:

$$
D=M+\frac{\sum_{j=1}^{M} \lambda_{j}}{\left|\lambda_{M+1}\right|}
$$

where $\mathrm{M}$ is the largest integer for which $\sum_{j=1}^{M} \lambda_{j}>0$ and $\sum_{j=1}^{M+1} \lambda_{j}<0$.

\subsection{Invariance and fixed points}

System (5) is invariant under the transformation

$$
(\mathbf{x}, \mathbf{y}, \mathbf{z}, \mathbf{u}, \mathbf{v}, \mathbf{w}) \longrightarrow(-\mathbf{x},-\mathbf{y}, \mathbf{z},-\mathbf{u},-\mathbf{v},-\mathbf{w}) .
$$

This means that if $(\mathbf{x}, \mathbf{y}, \mathbf{z}, \mathbf{u}, \mathbf{v}, \mathbf{w})$ is a solution of the system $(5)$ then $(-\mathbf{x},-\mathbf{y}, \mathbf{z},-\mathbf{u},-\mathbf{v},-\mathbf{w})$ is also a solution.

The fixed points of the system (5) can be found by solving the equations ${ }_{c} D_{t}^{\alpha} \mathbf{x}=0,{ }_{c} D_{t}^{\alpha} \mathbf{y}=$ $0,{ }_{c} D_{t}^{\alpha} \mathbf{z}=0,{ }_{c} D_{t}^{\alpha} \mathbf{u}=0,{ }_{c} D_{t}^{\alpha} \mathbf{v}=0$ and ${ }_{c} D_{t}^{\alpha} \mathbf{w}=0$. When $a b d h k \neq 0$ system (5) has one isolated fixed point $E_{0}=(0,0,0,0,0,0)$.

The Jacobian matrix of the system (5) at $E_{0}$ is

$$
A=\left(\begin{array}{cccccc}
-a & a & 0 & 1 & 0 & 0 \\
c & -1 & 0 & 0 & 1 & 0 \\
0 & 0 & -b & 0 & 0 & 0 \\
0 & 0 & 0 & d & 0 & 0 \\
0 & -k & 0 & 0 & 0 & 0 \\
0 & l & 0 & 0 & 0 & h
\end{array}\right)
$$

The characteristic polynomial of $A$ at the fixed point $E_{0}$ is

$$
(\lambda+b)(\lambda-d)(\lambda-h)\left[\lambda^{3}+(a+1) \lambda^{2}+(a+k-a c) \lambda+a k\right]=0 .
$$

According to Routh-Hurwitz conditions all roots $\lambda$ of (7) are negative real parts if

$$
b>0, \quad d<0, \quad h<0, \quad a>-1, \quad k>a(a+1)(c-1) \text { and } a k>0 .
$$

Then $E_{0}$ is stable. In fact, the conditions in (8) are sufficient but not necessary. To investigate the necessary and sufficient stability condition we note that from the stability theory of fractional-order systems, when all eigenvalues of (7) lie inside angular sector $|\arg (\lambda(A))|>\alpha \pi / 2$ then the fractional-order system is stable.

Theorem 1 [44]: The following autonomous system:

$$
{ }_{c} D_{t}^{\alpha} \mathbf{x}=A \mathbf{x}, \quad \mathbf{x}(0)=\mathbf{x}_{0},
$$

with $0<\alpha<1, \mathbf{x} \in \mathbf{R}^{n}$ and $A \in \mathbf{R}^{n \times n}$, is asymptotically stable if and only if $|\arg (\lambda(A))|>$ $\alpha \pi / 2$ is satisfied for all eigenvalues $\lambda$ of matrix $A$. 
3.2 Fixing $(a, b, c, d, k, h, l)=(10,8 / 3,28,2,65,3,5)$, and varying the fractional-order $\alpha$

In this subsection, we calculated the values of the fractional-order $\alpha$ at which our system (5) has hyperchaotic behavior. Fix the system parameters $(a, b, c, d, k, h, l)$ and varying the fractionalorder such that $\alpha \in[0.6,1]$ with the initial values of state variables as $(3,5,9,13,0,15)$. The Lyapunov exponents of (5) versus $\alpha$ are depicted in Figs. 1(a-c). These figures, shows that system (5) has hyperchaotic attractors of order 2 where $\alpha \in[0.6,1]$, when $\alpha \in[0.6,0.7)$ and $[0.8,0.83)$ system $(5)$ has hyperchaotic attractors of order 3 , while it has hyperchaotic attractors of order 2 when $\alpha \in[0.9,1)$. In Fig. 1(d) we plotted the Poincaré map of system (5) on the x-y plane. In Table 2, we calculated the Lyapunov exponents and Lyapunov dimension of system (5) with different values of the fractional-order. From this table, system (5) is a dissipative since the sum of its Lyapunov exponents is negative in each case. In Fig. 2 we plotted the projections of the hyperchaotic attractor of order 2 of system (5) with $\alpha=0.97$.

Table 2. Lyapunov exponents and Lyapunov dimension with $(a, b, c, d, k, h, l)=(10,8 / 3,28,2,65,3,5)$.

\begin{tabular}{ccccccccc}
\hline$\alpha$ & $\lambda_{1}$ & $\lambda_{2}$ & $\lambda_{3}$ & $\lambda_{4}$ & $\lambda_{5}$ & $\lambda_{6}$ & Lyapunov dimension & Dynamics \\
\hline 0.97 & 3.7061 & 0.0753 & -0.0165 & -0.0163 & -1.2107 & -13.3348 & 5.1903 & hyperchaotic of order 2 \\
0.93 & 4.9732 & 0.0744 & -0.0178 & -0.0154 & -1.5940 & -17.8812 & 5.1913 & hyperchaotic of order 2 \\
0.89 & 6.6630 & 0.0782 & -0.0084 & -0.0278 & -2.1033 & -23.9522 & 5.1921 & hyperchaotic of order 2 \\
0.84 & 9.5849 & 0.0671 & 0.0024 & -0.0360 & -2.9673 & -34.4682 & 5.1930 & hyperchaotic of order 3 \\
0.6 & 53.5456 & 7.2386 & 0.1000 & -0.5167 & -24.3904 & -194.3771 & 5.1851 & hyperchaotic of order 3 \\
\hline
\end{tabular}

\subsection{Fixing $(a, c, d, k, h, l)=(10,28,2,65,3,5), \alpha=0.93$ and varying $b$}

We varying the parameter $b$ such that $b \in(0,30]$. We found system (5) has hyperchaotic attractors of order 2 for $b \in[5,30]$, when $b \in(0,5)$ it has hyperchaotic attractors of order 3. The Lyapunov exponents and Lyapunov dimension of system (5) with different values of the parameter $b$ are calculated in Table 3. In Figs. 3(a) and 3(b) we plotted the Lyapunov exponents of system $(5)$ with $b \in(0,30]$ and the bifurcation diagram $(b, x)$ for $b \in[2,8]$ with $(a, c, d, k, h, l)=(10,28,2,65,3,5)$ and $\alpha=0.93$ respectively.

Table 3. Lyapunov exponents and Lyapunov dimension with $(a, c, d, k, h, l)=(10,28,2,65,3,5)$ and $\alpha=0.93$.

\begin{tabular}{|c|c|c|c|c|c|c|c|c|}
\hline$b$ & $\lambda_{1}$ & $\lambda_{2}$ & $\lambda_{3}$ & $\lambda_{4}$ & $\lambda_{5}$ & $\lambda_{6}$ & Lyapunov dimension & Dynamics \\
\hline$\overline{1.7}$ & 4.9739 & 0.0350 & 0.0097 & -0.7247 & -1.9260 & -15.2165 & 5.1556 & hyperchaotic of order 3 \\
\hline 3.2 & 4.9576 & 0.3844 & 0.0829 & -0.0715 & -0.6744 & -20.0293 & 5.2336 & hyperchaotic of order 3 \\
\hline 4 & 4.9804 & 1.0275 & 0.5081 & -0.0309 & -3.4597 & -19.7121 & 5.1535 & hyperchaotic of order 3 \\
\hline 23 & 4.9710 & 0.0774 & -0.4495 & -4.1840 & -20.5319 & -28.2838 & 4.0202 & hyperchaotic of order 2 \\
\hline 29 & 4.9829 & 0.0323 & -1.8085 & -2.0152 & -19.7434 & -39.8292 & 4.0603 & hyperchaotic of order 2 \\
\hline
\end{tabular}

\subsection{Fixing $(a, b, c, d, h, l)=(10,8 / 3,28,2,3,5), \alpha=0.93$ and varying $k$}

Fix the system parameters $(a, b, c, q, h, l)$ and the fractional-order $\alpha$, and varying $k$ such as $k \in$ $(0,80]$. We obtained system (5) has hyperchaotic attractors of order 2 for $k \in[60,80)$, and it has hyperchaotic attractors of order 3 for $k \in[20,60)$, while it has hyperchaotic attractors of order 4 for $k \in[0.5,20)$, when $k \in(0,0.5)$ it has hyperchaotic attractors of order 5 . Table 4 shows the Lyapunov exponents and Lyapunov dimension of system (5) with different values of the parameter $k$. The Lyapunov exponents of system (5) versus $k \in(0,80]$ are depicted in Fig. 4(a) and in Fig. 4(b) we plotted bifurcation diagram $(k, x)$ for $k \in[60,65]$ with $(a, b, c, d, h, l)=$ $(10,8 / 3,28,2,3,5)$ and $\alpha=0.93$. In Fig. 5 we plotted the hyperchaotic attractor of order 5 of 


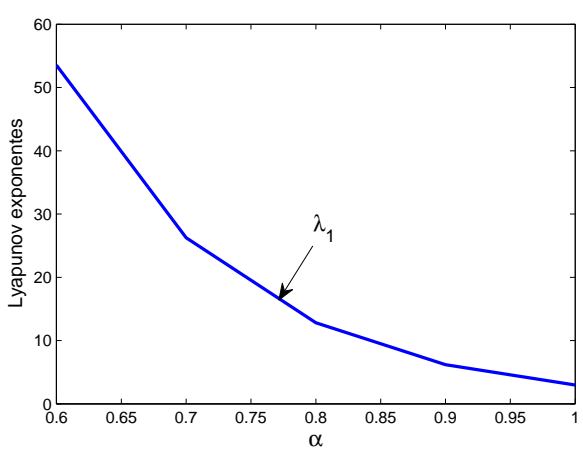

(a)

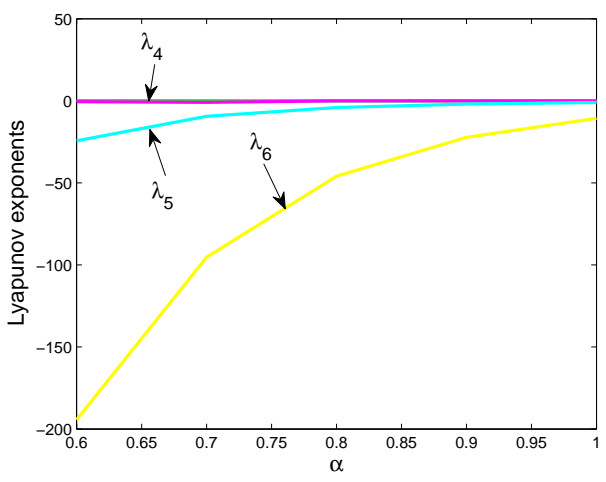

(c)

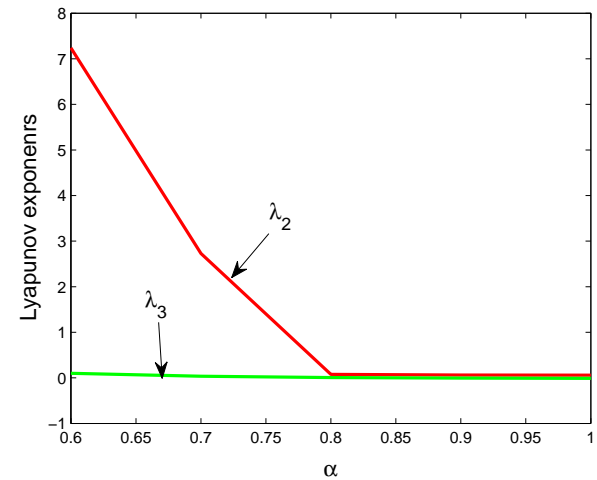

(b)

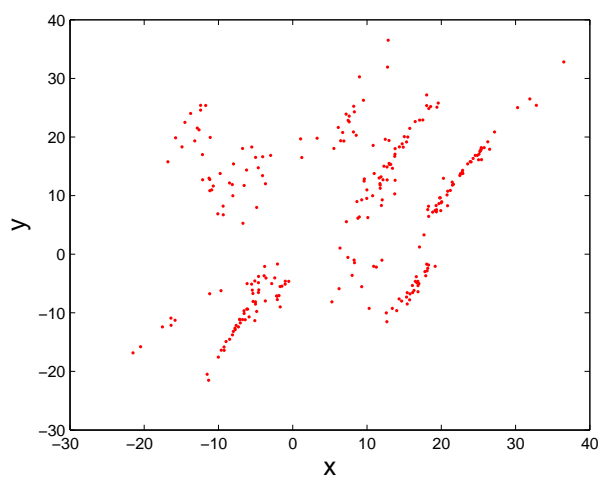

(d)

Figure 1: (a)-(c) Lyapunov exponents of system (5) with fractional-order where $\alpha \in[0.6,1]$ (d) Poincaré map of system (5) on the x-y plane with $(a, b, c, d, k, h, l)=(10,8 / 3,28,2,0.35,3,5)$ and $\alpha=0.93$. 


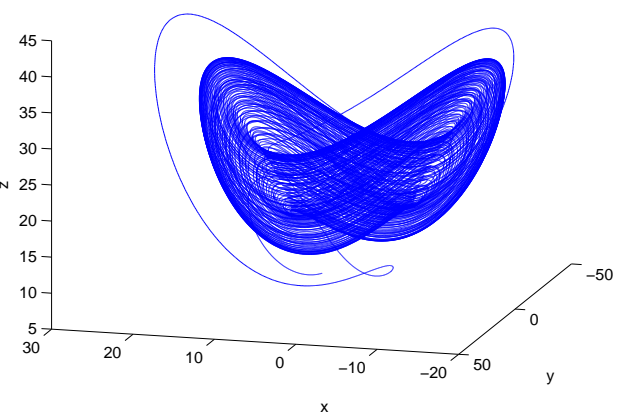

(a)

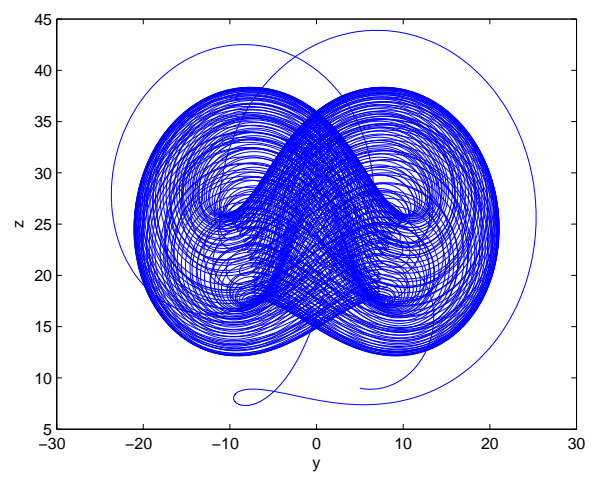

(c)

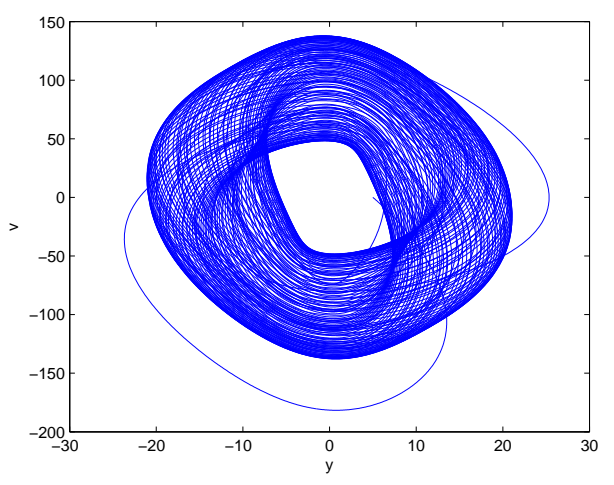

(e)

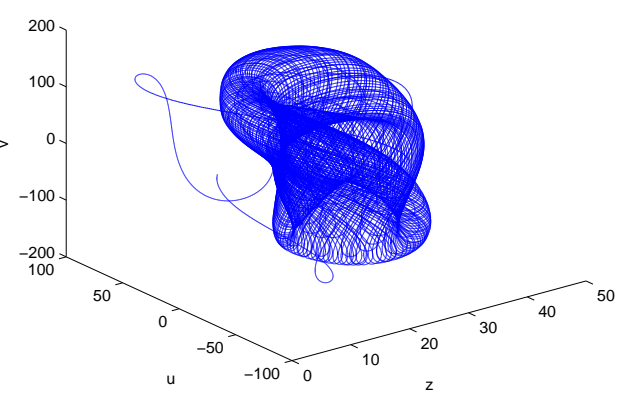

(b)

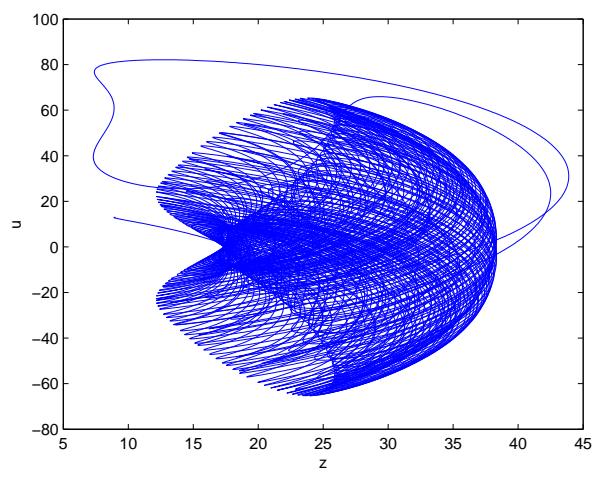

(d)

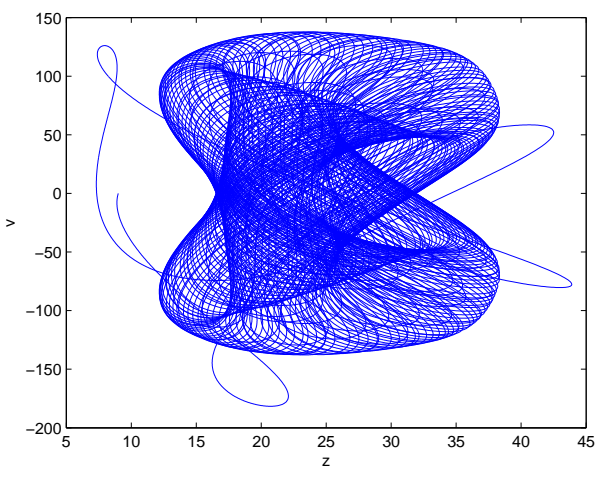

(f)

Figure 2: Hypercaotic attractor of order 2 of system (5) with $(a, b, c, d, k, h, l)=(10,8 / 3,28,2,65,3,5)$ and $\alpha=0.97$, on: (a) x-y-z space, (b) z-u-v space, (c) y-z plane, (d) z-u plane, (e) y-v plane, (f) z-v plane. 


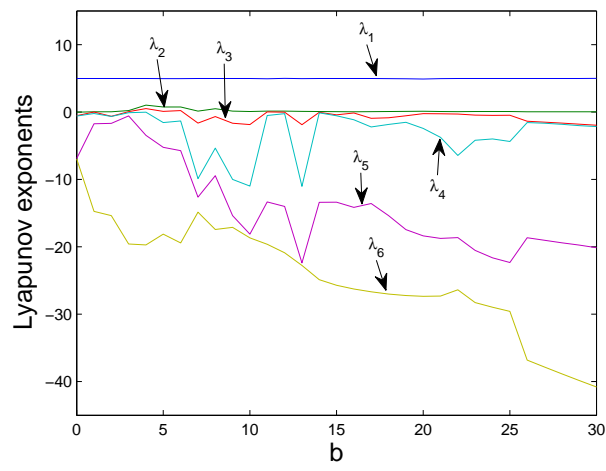

(a)

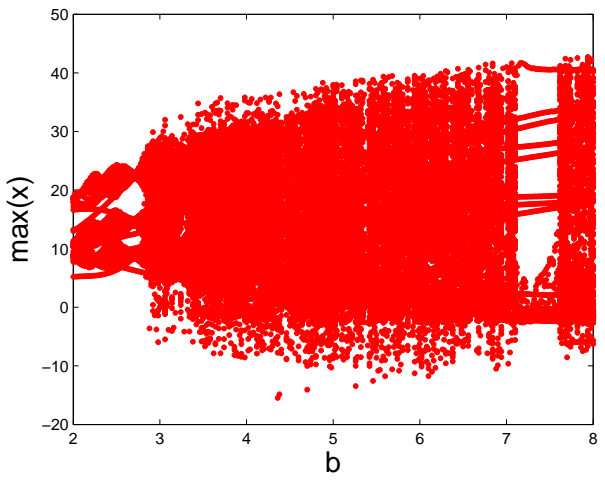

(b)

Figure 3: (a) Lyapunov exponents of system (5) vs $b$ where $b \in(0,30]$ (b) Bifurcation diagram of system (5) with $(a, c, d, k, h, l)=(10,28,2,65,3,5), \alpha=0.93$ and $b \in[2,8]$.

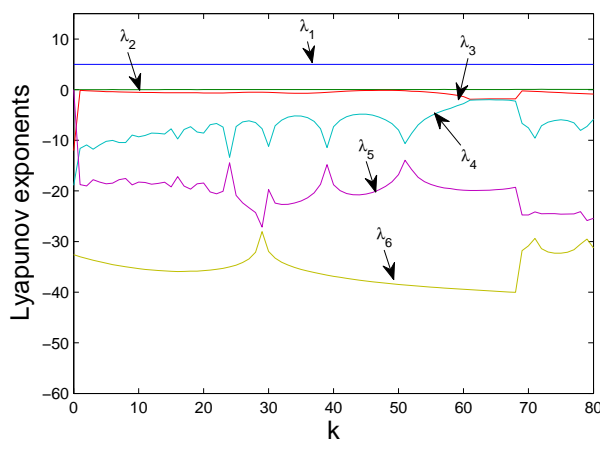

(a)

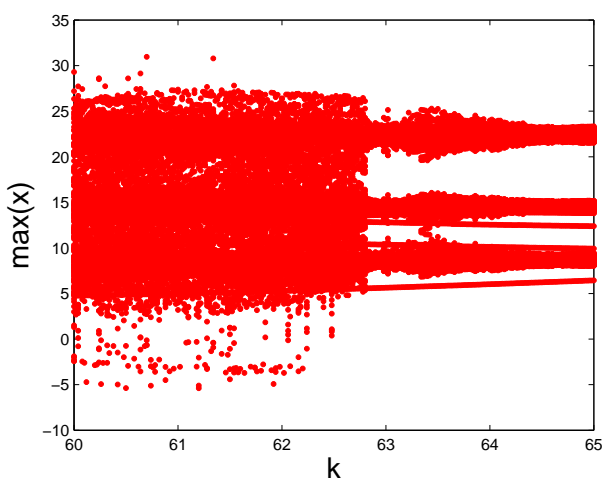

(b)

Figure 4: (a) Lyapunov exponents of system (5) vs $k$ where $k \in(0,80]$ (b) Bifurcation diagram of system (5) with $(a, b, c, d, h, l)=(10,8 / 3,28,2,3,5), \alpha=0.93$ and $k \in[60,65]$.

system (5) in different projections with $k=0.35$.

Table 4. Lyapunov exponents and Lyapunov dimension with $(a, b, c, d, h, l)=(10,8 / 3,28,2,3,5)$ and $\alpha=0.93$.

\begin{tabular}{ccccccccc}
\hline$k$ & $\lambda_{1}$ & $\lambda_{2}$ & $\lambda_{3}$ & $\lambda_{4}$ & $\lambda_{5}$ & $\lambda_{6}$ & Lyapunov dimension & Dynamics \\
\hline 0.1 & 4.9690 & 0.8295 & 0.4909 & 0.0062 & 0.0040 & -21.1384 & 5.2980 & hyperchaotic of order 5 \\
0.35 & 4.9687 & 1.0374 & 0.6179 & 0.0020 & 0.0096 & -21.0957 & 5.3145 & hyperchaotic of order 5 \\
3 & 4.9626 & 1.1803 & 0.4338 & 0.0526 & -0.0057 & -21.0847 & 5.3141 & hyperchaotic of order 4 \\
25 & 4.9783 & 0.4932 & 0.2328 & -0.0698 & -0.6171 & -19.4772 & 5.2576 & hyperchaotic of order 3 \\
67 & 4.9733 & 0.0414 & -0.0640 & -0.0683 & -2.0018 & -17.3418 & 5.1661 & hyperchaotic of order 2 \\
\hline
\end{tabular}

\subsection{Clarify the degree of chaos between systems (4) and (5)}

In this subsection we compare between the integer order system (4) and its fractional version (5) of degree of chaos.

Yang et al. [40] calculated Lyapunov exponents of system (4) with $a=10, b=8 / 3, c=$ $28, d=2, h=l=1$ and varying $k$, the results are shown in Table 5 below 


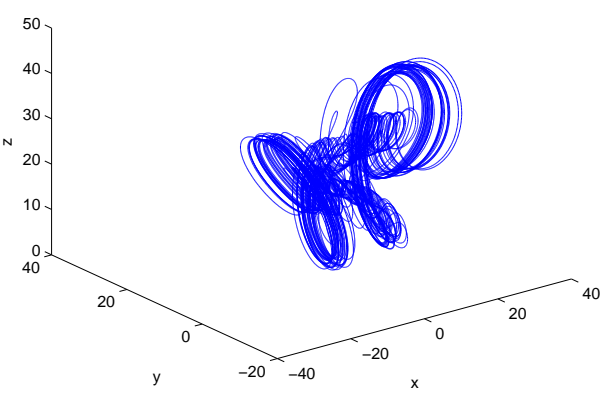

(a)

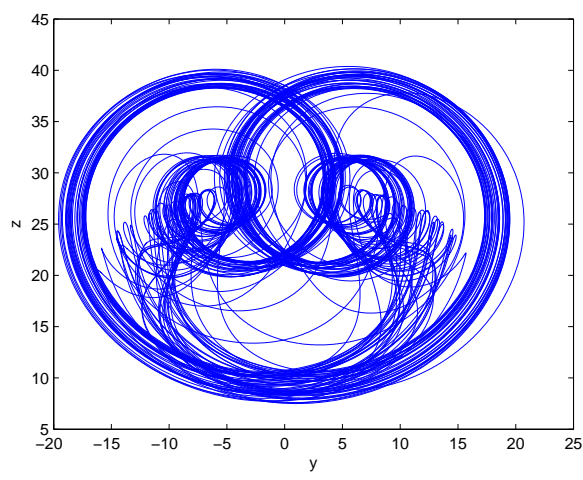

(c)

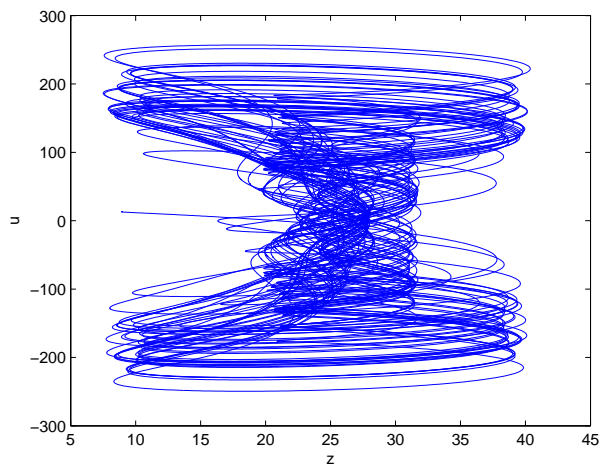

(e)

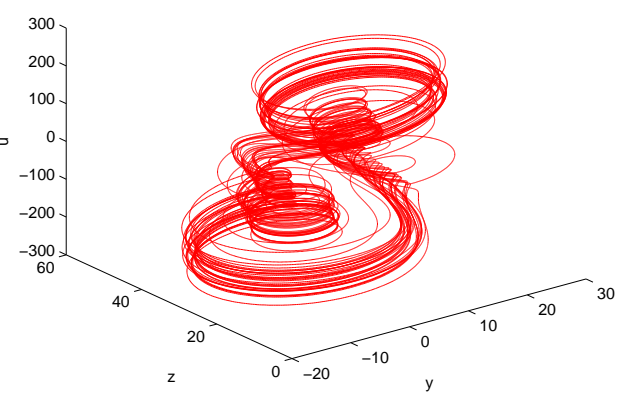

(b)

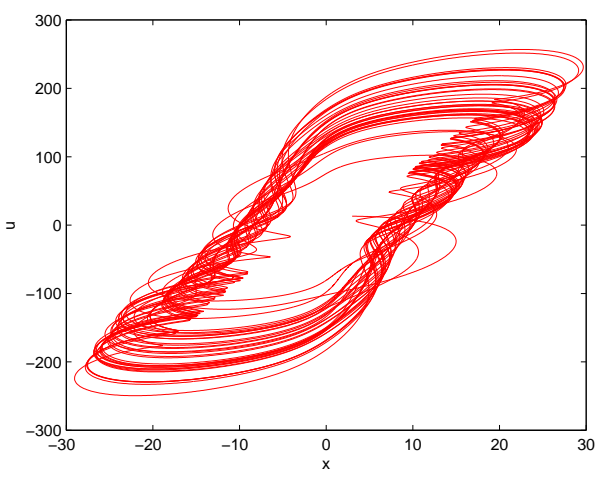

(d)

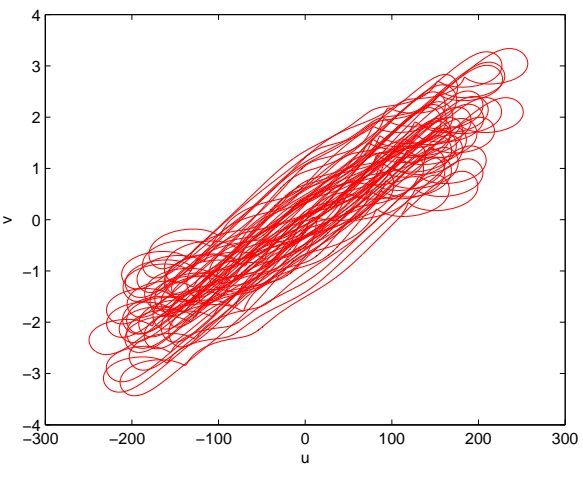

(f)

Figure 5: Hyperchaotic attractor of order 5 of system (5) with $(a, b, c, d, h, l)=(10,8 / 3,28,2,3,5), \alpha=0.93$ and $k=0.35$. 
Table 5. Lyapunov exponents of system (4) with $(a, b, c, d, h, l)=(10,8 / 3,28,2,1,1)$

\begin{tabular}{cccccccc}
\hline$k$ & $\lambda_{1}$ & $\lambda_{2}$ & $\lambda_{3}$ & $\lambda_{4}$ & $\lambda_{5}$ & $\lambda_{6}$ & Dynamics \\
\hline 7.3 & 0.9995 & 0.5021 & 0.3251 & 0.0557 & 0.0000 & -12.5491 & hyperchaotic of order 4 \\
44.8 & 0.9998 & 0.4981 & 0.2550 & 0.0000 & -0.7938 & -11.6275 & hyperchaotic of order 3 \\
60.5 & 0.9996 & 0.2746 & 0.0000 & -0.0286 & -0.7673 & -11.1450 & hyperchaotic of order 2 \\
63.3 & 0.9997 & 0.0007 & -0.0369 & -0.0388 & -0.2628 & -11.3285 & chaotic
\end{tabular}

While, we calculated the Lyapunov exponents of system (5) with the same values of the system parameters, $\alpha=0.84$ and varying $k$, the results are shown in Table 6 below

Table 6. Lyapunov exponents of system (4) with $(a, b, c, d, h, l)=(10,8 / 3,28,2,1,1)$ and $\alpha=0.84$.

\begin{tabular}{cccccccc}
\hline$k$ & $\lambda_{1}$ & $\lambda_{2}$ & $\lambda_{3}$ & $\lambda_{4}$ & $\lambda_{5}$ & $\lambda_{6}$ & Dynamics \\
\hline 7.3 & 2.7932 & 1.4184 & 0.8048 & 0.1177 & 0.1509 & -39.5041 & hyperchaotic of order 5 \\
44.8 & 2.1053 & 1.9826 & 0.9717 & 0.3459 & -1.8993 & -37.7213 & hyperchaotic of order 4 \\
60.5 & 1.9183 & 0.9958 & 0.3592 & -0.2766 & -2.0070 & -35.2155 & hyperchaotic of order 3 \\
63.3 & 1.9197 & 0.3659 & 0.3473 & -0.5000 & -2.5626 & -33.7996 & hyperchaotic of order 2 \\
\hline
\end{tabular}

It's clear from these two tables that, the fractional version (5) is more chaotic than the integer one (4). This is an additional characteristic of the new fractional-order system.

\section{Synchronization of hyperchaotic attractors of system (5)}

In this section, we study the synchronization of two identical 6D fractional-order hyperchaotic of order 5 of system (5) using active control technique with drive, response and error systems, defined as:

$$
\begin{gathered}
{ }_{c} D_{t}^{\alpha} \mathbf{x}(t)=A \mathbf{x}(t)+f(\mathbf{x}(t)), \\
{ }_{c} D_{t}^{\alpha} \mathbf{y}(t)=A \mathbf{y}(t)+f(\mathbf{y}(t))+\vartheta, \\
e(t)=\mathbf{y}(t)-\mathbf{x}(t),
\end{gathered}
$$

where $\mathbf{x}=\left(\mathbf{x}_{1}, \mathbf{x}_{3}, \ldots, \mathbf{x}_{6}\right)^{T}, \mathbf{y}=\left(\mathbf{y}_{1}, \mathbf{y}_{3}, \ldots, \mathbf{y}_{6}\right)^{T}$ are the state vectors of systems (9), (10), $A \in \mathbf{R}^{6 \times 6}$ is constant matrix of system parameters, $f: \mathbf{R}^{6} \longrightarrow \mathbf{R}^{6}$ is continuous vector function, $\vartheta: \mathbf{R}^{6} \times \mathbf{R}^{6} \longrightarrow \mathbf{R}^{6}, \vartheta=\left(\vartheta_{1}, \vartheta_{2}, \ldots, \vartheta_{6}\right)^{T}$ is control function of the response system (10) and $\mathrm{T}$ denotes the transpose.

Theorem 2: Synchronization between two identical hyperchaotic 6-dimensional fractionalorder dynamical systems (9), (10) and the error in the form (11) will be achieved if the control functions are designed as follows:

$$
\vartheta=f(x)-f(y)-K e,
$$

where $K$ is the control gain matrix, which satisfies $|\arg (\lambda(A-K))|>\alpha \pi / 2$ for all eigenvalues of $(A-K)$.

proof : The $\alpha$-derivative of the synchronization error (11) is

$$
{ }_{c} D_{t}^{\alpha} e(t)={ }_{c} D_{t}^{\alpha} \mathbf{y}(t)-{ }_{c} D_{t}^{\alpha} \mathbf{x}(t)
$$

Substituting Eqs. (9), (10) and (12) in Eq. (13) then

$$
{ }_{c} D_{t}^{\alpha} e(t)=(A-K) e .
$$


From stability theory of fractional-order system and since $|\arg (\lambda(A-K))|>\alpha \pi / 2$ then the error vector $e(t)$ is asymptotically stable, which means that $\lim _{t \rightarrow \infty}\|e(t)\|=0$.

The drive and the response systems for hyperchaotic $6 \mathrm{D}$ fractional-order system (5) can be written as:

$$
\begin{gathered}
\left(\begin{array}{c}
{ }_{c} D_{t}^{\alpha} \mathbf{x}_{1} \\
{ }_{c} D_{t}^{\alpha} \mathbf{y}_{1} \\
{ }_{c} D_{t}^{\alpha} \mathbf{z}_{1} \\
{ }_{c} D_{t}^{\alpha} \mathbf{u}_{1} \\
{ }_{c} D_{t}^{\alpha} \mathbf{v}_{1} \\
{ }_{c} D_{t}^{\alpha} \mathbf{w}_{1}
\end{array}\right)=\left(\begin{array}{cccccc}
-a & a & 0 & 1 & 0 & 0 \\
c & -1 & 0 & 0 & 1 & 0 \\
0 & 0 & -b & 0 & 0 & 0 \\
0 & 0 & 0 & d & 0 & 0 \\
0 & -k & 0 & 0 & 0 & 0 \\
0 & l & 0 & 0 & 0 & h
\end{array}\right)\left(\begin{array}{c}
\mathbf{x}_{1} \\
\mathbf{y}_{1} \\
\mathbf{z}_{1} \\
\mathbf{u}_{1} \\
\mathbf{v}_{1} \\
\mathbf{w}_{1}
\end{array}\right)+\left(\begin{array}{c}
0 \\
-\mathbf{x}_{1} \mathbf{z}_{1} \\
\mathbf{x}_{1} \mathbf{y}_{1} \\
-\mathbf{x}_{1} \mathbf{z}_{1} \\
0 \\
0
\end{array}\right), \\
\left(\begin{array}{c}
{ }_{c} D_{t}^{\alpha} \mathbf{x}_{2} \\
{ }_{c} D_{t}^{\alpha} \mathbf{y}_{2} \\
{ }_{c} D_{t}^{\alpha} \mathbf{z}_{2} \\
{ }_{c} D_{t}^{\alpha} \mathbf{u}_{2} \\
{ }_{c} D_{t}^{\alpha} \mathbf{v}_{2} \\
{ }_{c} D_{t}^{\alpha} \mathbf{w}_{2}
\end{array}\right)=\left(\begin{array}{cccccc}
-a & a & 0 & 1 & 0 & 0 \\
c & -1 & 0 & 0 & 1 & 0 \\
0 & 0 & -b & 0 & 0 & 0 \\
0 & 0 & 0 & d & 0 & 0 \\
0 & -k & 0 & 0 & 0 & 0 \\
0 & l & 0 & 0 & 0 & h
\end{array}\right)\left(\begin{array}{c}
\mathbf{x}_{2} \\
\mathbf{y}_{2} \\
\mathbf{z}_{2} \\
\mathbf{u}_{2} \\
\mathbf{v}_{2} \\
\mathbf{w}_{2}
\end{array}\right)+\left(\begin{array}{c}
0 \\
-\mathbf{x}_{2} \mathbf{z}_{2} \\
\mathbf{x}_{2} \mathbf{y}_{2} \\
-\mathbf{x}_{2} \mathbf{z}_{2} \\
0 \\
0
\end{array}\right)+\left(\begin{array}{c}
\vartheta_{1} \\
\vartheta_{2} \\
\vartheta_{3} \\
\vartheta_{4} \\
\vartheta_{5} \\
\vartheta_{6}
\end{array}\right) .
\end{gathered}
$$

The values of the systems parameters can be taken as: $(a, b, c, d, k, h, l)=(10,8 / 3,28,2,0.35,3,5)$. If we choose the control gain matrix $\mathrm{K}$ such that

$$
K=\left(\begin{array}{cccccc}
0 & 0 & 0 & 0 & 0 & 0 \\
28 & 0 & 0 & 0 & 0 & 0 \\
0 & 0 & 0 & 0 & 0 & 0 \\
0 & 0 & 0 & 4 & 0 & 0 \\
0 & -65 & 0 & 0 & 1 & 0 \\
0 & 5 & 0 & 0 & 0 & 9
\end{array}\right)
$$

Then, we have $|\arg (\lambda(A-K))|>\alpha \pi / 2$. From Eq. (12) the control functions can be obtained as:

$$
\left(\begin{array}{c}
\vartheta_{1} \\
\vartheta_{2} \\
\vartheta_{3} \\
\vartheta_{4} \\
\vartheta_{5} \\
\vartheta_{6}
\end{array}\right)=\left(\begin{array}{c}
0 \\
\mathbf{x}_{2} \mathbf{z}_{2}-\mathbf{x}_{1} \mathbf{z}_{1}-28\left(\mathbf{x}_{2}-\mathbf{x}_{1}\right) \\
\mathbf{x}_{1} \mathbf{y}_{1}-\mathbf{x}_{2} \mathbf{y}_{2} \\
\mathbf{x}_{2} \mathbf{z}_{2}-\mathbf{x}_{1} \mathbf{z}_{1}-4\left(\mathbf{u}_{2}-\mathbf{u}_{1}\right) \\
65\left(\mathbf{y}_{2}-\mathbf{y}_{1}\right)-\left(\mathbf{v}_{2}-\mathbf{v}_{1}\right) \\
-5\left(\mathbf{y}_{2}-\mathbf{y}_{1}\right)-9\left(\mathbf{w}_{2}-\mathbf{w}_{1}\right)
\end{array}\right)
$$

Systems (15) and (16) with (18) are solved numerically using Adams-Bashforth-Moulton method [41] for $\alpha=0.93$ and $(a, b, c, d, k, h, l)=(10,8 / 3,28,2,0.35,3,5)$. Numerical simulation results are illustrated in Figs. 6 and 7. In Fig. 6 the solutions of (15) and (16) with (18) are plotted subject to the initial conditions $\mathbf{x}(0)=(3,5,9,13,0,15)^{T}$ and $\mathbf{y}(0)=(12.7621,15.9,16.6769,1,2,2)^{T}$. It is clearly shown that synchronization is achieved. In Fig. 7 it can be seen that synchronization errors $e_{j}, j=1,2, \ldots, 6$ converge to zero. 


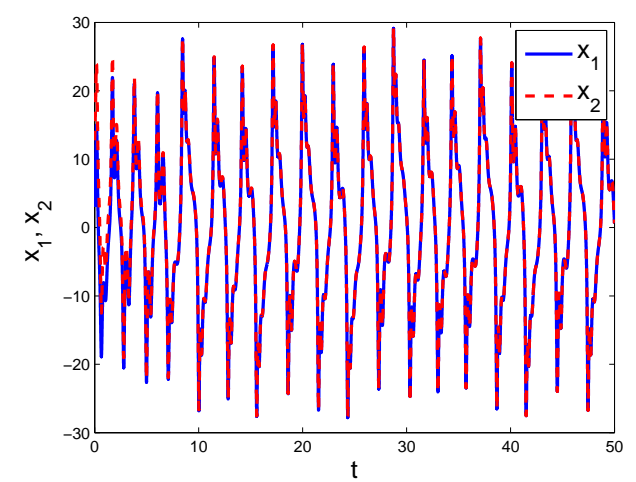

(a)

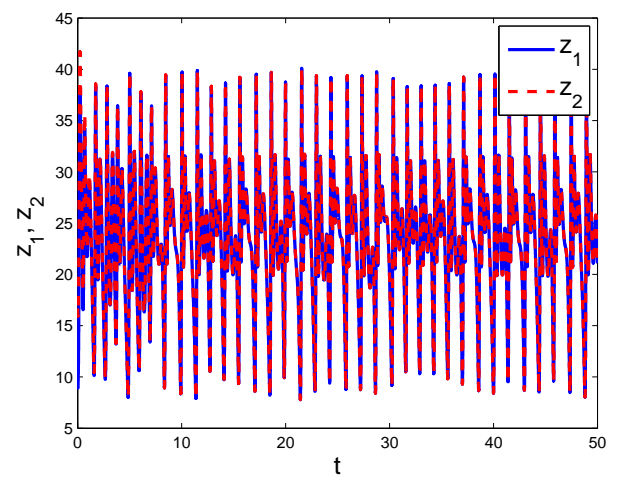

(c)

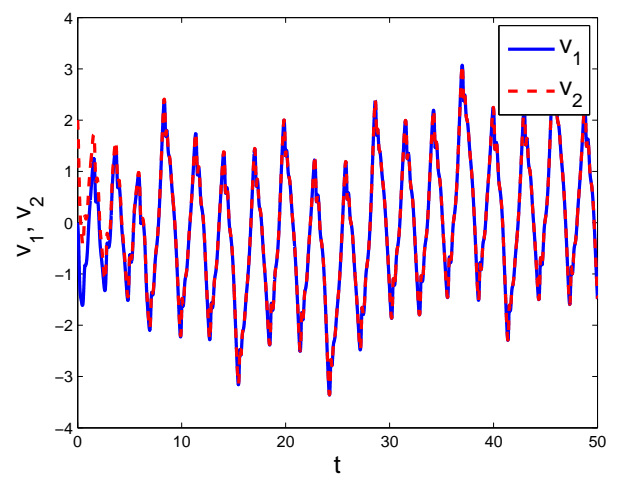

(e)

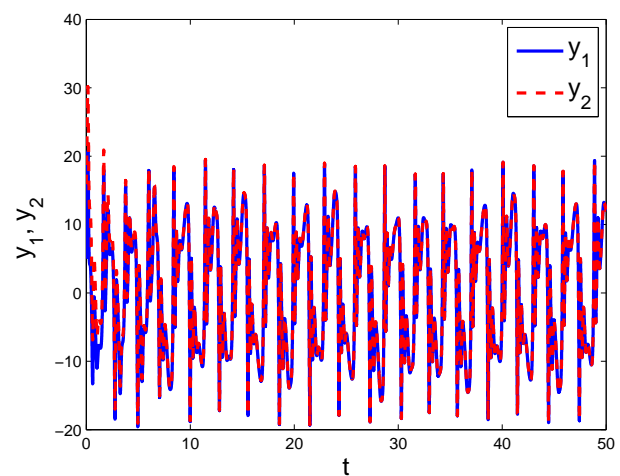

(b)

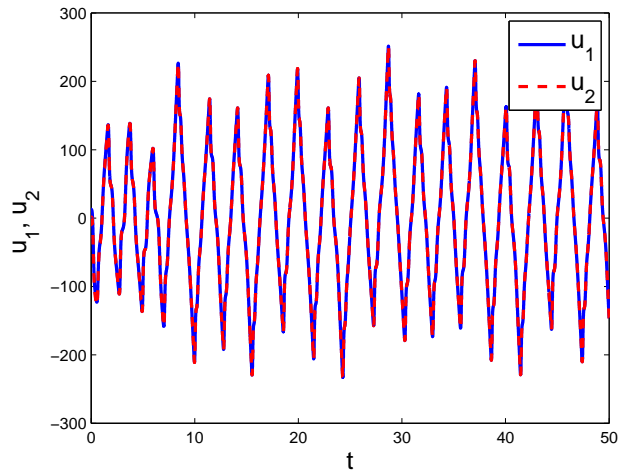

(d)

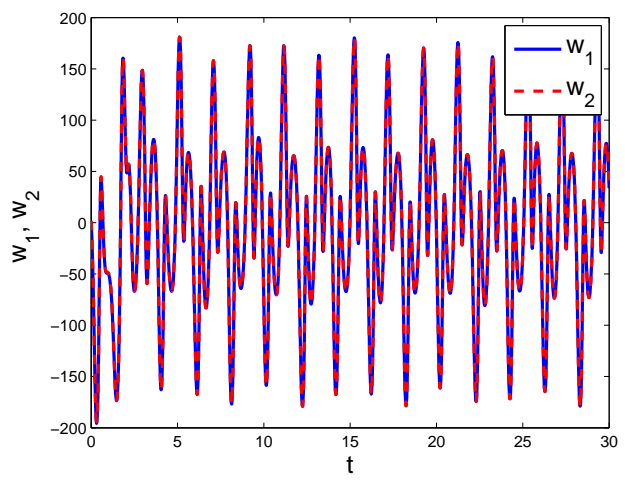

(f)

Figure 6: Synchronization of hyperchaotic attractor of order 5 of of systems (15) and (16) with $\alpha=0.93$ and $(a, b, c, d, k, h, l)=(10,8 / 3,28,2,0.35,3,5)$. 


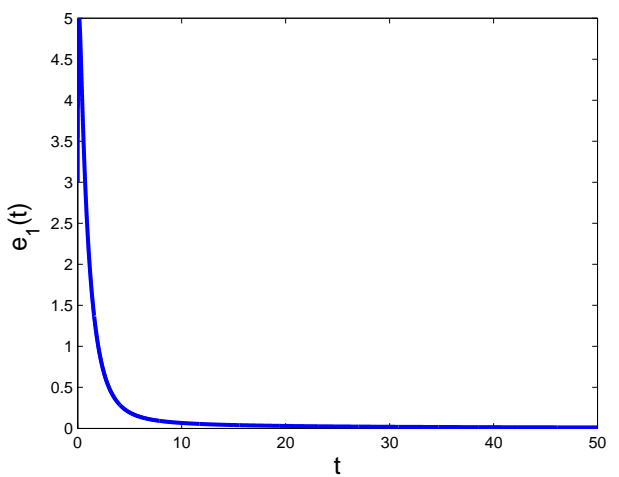

(a)

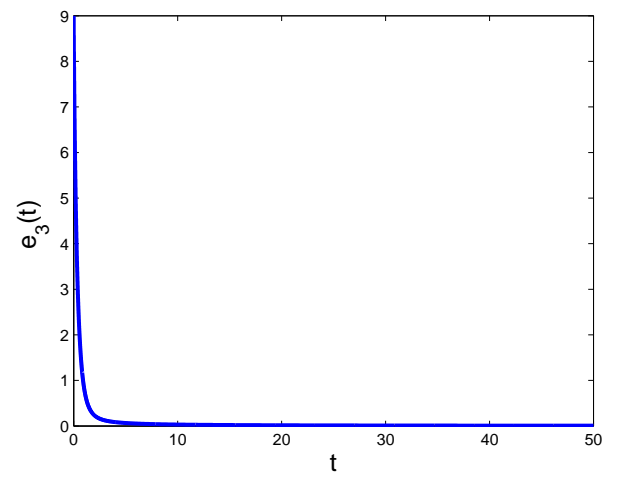

(c)

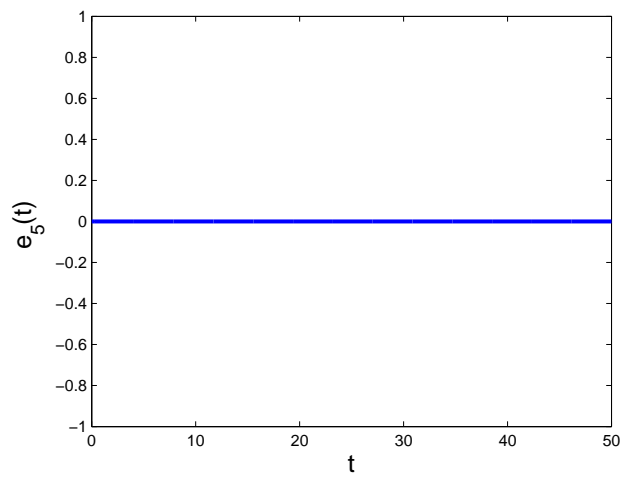

(e)

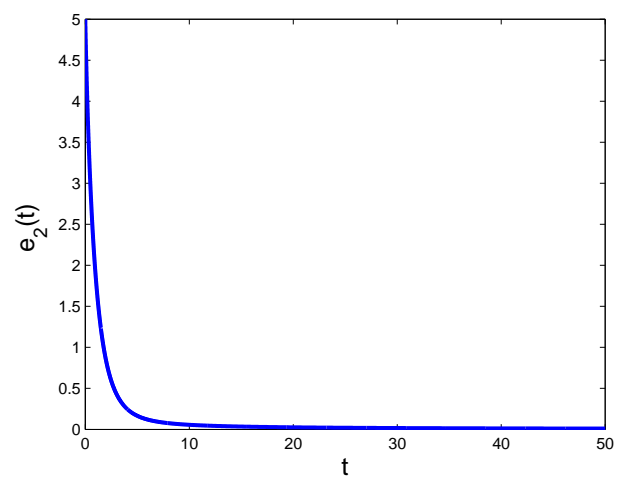

(b)

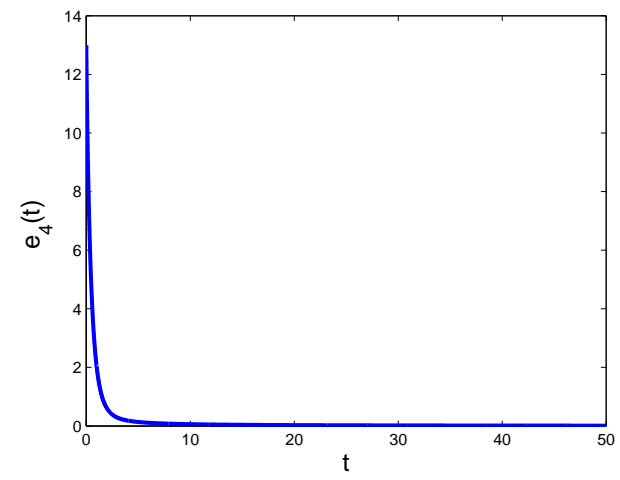

(d)

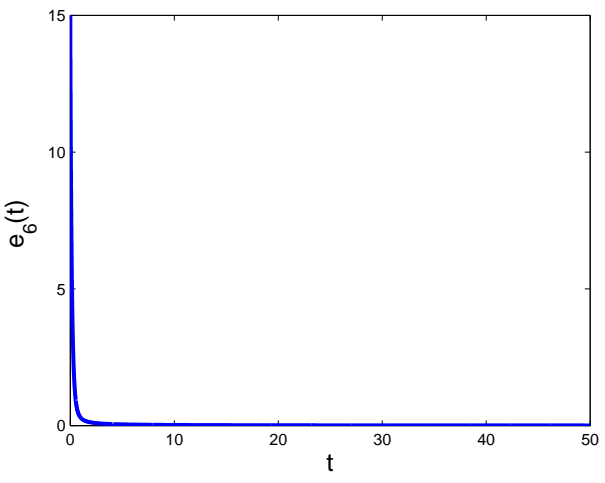

(f)

Figure 7: The dynamics of error evolution described by the solutions of system (14). 


\section{Conclusion}

In this paper, the fractional version of the new $6 \mathrm{D}$ Lorenz-like system is proposed. The new fractional-order system (5) generates hyperchaotic attractors of order 5 as shown in Fig. 5. This kind of hyperchaotic attractors doesn't exist for system (4) with integer order. The new hyperchaotic system has a unique fixed point when abdhk $\neq 0$. The stability region of the fractional-order system (5) is investigated. We calculated the values of the fractional-order $\alpha$ and the system parameters $a, b, c, d, k, h$ and $l$ at which hyperchaotic attractors of order 2 , 3,4 and 5 are exist. These calculations are based on the sign of the Lyapunov exponents. The bifurcation diagrams of system (5) plotted in Figs. 3(b) and 4(b). We used the Lyapunov exponents to compute the fractional Lyapunov dimension. An active control technique is used to study the complete synchronization between two identical 6D fractional-order hyperchaotic of order 5 of system (5). The analytical formula (18) of the control vector function is used to achieve the complete synchronization as shown in Fig. 6, while the synchronization errors go to zero as in Fig. 7. The numerical experiments are used to test the validity of analytical calculations, and good agrement is found. In communications using hyperchaotic attractors of higher order increases randomness and unpredictability of the system.

\section{Acknowledgment}

The authors thank the referees for very useful suggestions, which helped us improve considerably the presentation of our results. Also we would like to thank Prof. G.M. Mahmoud for his critical reading and review of manuscript.

\section{References}

[1] G.Y. Wang, X. Zhang, Y. Zheng, Y.X. Li, A new modified hyperchaotic Lü system, Physica A 371 (2006) 260-272.

[2] X. Wang, M. Wang, A hyperchaos generated form Lorenz system, Physica A 387 (2008) $3751-3758$.

[3] E.N. Lorenz, Deterministic non-periodic flow, J. Atmospheric Sci. 20 (1963) 130-141.

[4] O.E. Rössler, An equation for hyperchaos, Phys. Lett. A 71 (1979) 155-157.

[5] G. Chen, T. Ueta, Yet another chaotic attractor, Int. J. Bifurc. Chaos 9 (1999) 1465-1466.

[6] J.H. Lü, G.R. Chen, A new chaotic attractor coined, Int. J. Bifurc. Chaos 12 (2002) 659-661.

[7] Y. Xu, Y. Li, D. Liu, Response of fractional oscillators with viscoelastic term under random excitation, J. Comput. Nonlinear Dynamics 9 (2014) 031015-1.

[8] D. Liu, J. Li, Y. Xu, Principal resonance responses of SDOF systems with small fractional derivative damping under narrow-band random parametric excitation, Commun. Nonlinear Sci. Numer. Simulat 19 (2014) 3642-3652. 
[9] Y. Xu, R. Gu, H. Zhang D. Li, Chaos in Diffusionless Lorenz System with a Fractional Order and Its Control, Int. J. Bifurcation Chaos 22 (2012) 1250088.

[10] V.E. Arkhincheev, Anomalous diffusion in inhomogeneous media: Some exact results, Modeling Meas. Control A 26 (1993) 11-29.

[11] A.M.A. El-Sayed, Fractional order diffusion wave equation, Int. J. Theoret. Phys. 35 (1996) 311-322.

[12] G. Chen, G. Friedman, An RLC interconnect model based on Fourier analysis, IEEE Trans. Comput. Aided Des. Integr. Circuits Syst. 24 (2005) 170-183.

[13] V.G. Jenson, G.V. Jeffreys, Mathematical Methods in Chemical Engineering, 2nd ed., Academic Press, New York, 1977.

[14] K.S. Cole, Electric conductance of biological systems, in: Proc. Cold Spring Harbor Symp. Quant. Biol., Cold Spring Harbor, New York, (1993) 107-116.

[15] N. Laskin, Fractional market dynamics, Physica A 287 (2000) 482-492.

[16] R. Hilfer, Applications of Fractional Calculus in Physics, World Scientific Pub Co., Singapore, 2000.

[17] I. Podlubny, Fractional Differential Equations, Academic Press, San Diego, 1999.

[18] G.M. Mahmoud, M.E. Ahmed, T.M. Abed-Elhameed, Active control technique of fractional-order chaotic complex systems, Eur. Phys. J. Plus 131 (2016).

[19] Y. Xu, H. Wang, D. Liu, H. Huang, Sliding mode control of a class of fractional chaotic systems in the presence of parameter perturbations, J. Vib. Control 21 (2015) 435-448.

[20] Y. Xu, Y. Li, D. Liu, W. Jia, H. Huang, Responses of Duffing oscillator with fractional damping and random phase, Nonlinear Dyn. 74 (2013) 745-753.

[21] T. Liu, W. Xu, Y. Xu, Q. Han, Long-Term dynamics of autonomous fractional differential equations, Int. J. Bifurcation Chaos 26 (2016) 1650055.

[22] Y. Xu, Y. Li, D. Liu, A method to stochastic dynamical systems with strong nonlinearity and fractional damping, Nonlinear Dyn. 63 (2016) 2311-2321.

[23] W. Ahmad, R. El-Khazali, A. El-Wakil, Fractional order Wien bridge oscillator, Electron. Lett. 37 (2001) 1110-1112.

[24] Y. Wang, C. Li, Does the fractional Brusselator with efficient dimension less than 1 have a limit cycle? Phys. Lett. A 363 (2007) 414-419.

[25] X. Gao, J.B. Yu, Chaos in the fractional order periodically forced complex Duffing's oscillators, Chaos Solitons Fractals 24 (2005) 1097-1104.

[26] T.T. Hartley, C.F. Lorenzo, H.K. Qammer, Chaos in a fractional order Chua's system, IEEE Trans. Circuits Syst. 42 (1995) 485-490. 
[27] W.M. Ahmad, J.C. Sprott, Chaos in fractional order autonomous nonlinear systems, Chaos Solitons Fractals 16 (2003) 339-351.

[28] I. Grigorenko, E. Grigorenko, Chaotic dynamics of the fractional Lorenz system, Phys. Rev. Lett. 91 (2003) 034101.

[29] J.G. Lu, Chaotic dynamics of the fractional order Lü system and its synchronization, Phys. Lett. A 354 (2006) 305-311.

[30] C. Li, G. Chen, Chaos in the fractional order Chen system and its control, Chaos Solitons Fractals 22 (2004) 549-554.

[31] C. Li, G. Chen, Chaos and hyperchaos in the fractional order Rössler equations, Physica A 341 (2004) 55-61.

[32] X. Wu, Y. Lu, Generalized projective synchronization of the fractional-order Chen hyperchaotic system, Nonlinear Dyn. 57 (2009) 25-35.

[33] Y.G. Yu, H.X. Li, The synchronization of fractional-order Rössler hyperchaotic systems, Physica A 387 (2008) 1393-1403.

[34] Y. Xu, H. Wang, Y. Li, B. Pei, Image encryption based on synchronization of fractional chaotic systems, Commun. Nonlinear Sci. Numer. Simulat 19 (2014) 3735-3744.

[35] C.D. Li, X.F. Liao, Complet and lag synchronization of hyperchaotic system using small implulses, Chaos Solitons Fractals 22 (2004) 857-867.

[36] G.M. Mahmoud, E.E. Mahmoud, Lag synchronization of hyperchaotic complex nonlinear systems, Nonlin. Dyn. 67 (2012) 1613-1622.

[37] G.M. Mahmoud, E.E. Mahmoud, A.A. Arafa, On projective synchronization of hyperchaotic complex nonlinear systems based on passive theory for secure communications, Physica Scripta 87 (2013) 055002.

[38] G.M. Mahmoud, T.M. Abed-Elhameed, M.E. Ahmed, Generalization of combinationcombination synchronization of chaotic n-dimensional fractional-order dynamical systems, Nonlinear Dyn. 83 (2016) 1885-1893.

[39] H. Taghvafard, G.H. Erjaee, Phase and anti-phase synchronization of fractional order chaotic systems via active control, Commun. Nonlinear Sci. Numer. Simulat 16 (2011) 4079-4088.

[40] Q. Yang, W.M. Osman, C. Chen, A new 6D hyperchaotic system with four positive Lyapunov exponents coined, Int. J. Bifurc. Chaos 25 (2015) 1550060.

[41] K. Diethelm, N.J. Ford, A.D.Freed, A predictor-corrector approach for the numerical solution of fractional differential equations, Nonlinear Dyn. 29 (2002) 3-22.

[42] A. Wolf, J. Swift, H. Swinney, J. Vastano, Determining Lyapunov exponents from a time series, Physica D 16 (1985) 285-317. 
[43] G.M. Mahmoud, E.E. Mahmoud, M.E. Ahmed, On the hyperchaotic complex Lü system, Nonlinear Dyn. 58 (2009) 725-738.

[44] M.S. Tavazoei and M. Haeri, Chaotic attractors in incommensurate fractional order systems, Physica D 237 (2008) 2628-2637. 